\title{
Fairness and throughput enhancement based resource allocation scheme for underlay cognitive radio networks
}

\author{
KYRILLOS YOUSSEF*, NAGY MESSIHA and MOHAMMED ABD-ELNABY \\ Faculty of Electronic Engineering, Menoufia University, Menouf, Egypt \\ e-mail: kyrillos.fouad@gmail.com; Dr.nagy_wadie@hotmail.com; moh_naby@yahoo.com
}

MS received 17 July 2017; revised 21 December 2017; accepted 18 January 2018; published online 22 June 2018

\begin{abstract}
Acquiring good throughput and diminishing interference to primary users (PU) are the main objectives for secondary users in a cognitive radio (CR) network. This paper proposes a centralized subcarrier and power allocation scheme for underlay multi-user orthogonal frequency division multiplexing considering the rate loss and the interference those the PU can tolerate. The main purpose of the proposed scheme is to efficiently distribute the available subcarriers among cognitive users to enhance both the fairness and the throughput performance of the cognitive network while maintaining the QoS of primary users. Simulation results show that the proposed scheme achieves a significantly higher CR network throughput than that of the conventional interference power constraint (IPC) based schemes and provides a significantly enhanced fairness performance. Also, contrary to the conventional IPC based schemes, the proposed scheme is able to significantly increase the achieved throughput as the number of $\mathrm{CR}$ users increases.
\end{abstract}

Keywords. Cognitive radio; OFDM; resource allocation; spectrum sharing; interference constraints; fairness.

\section{Introduction}

As the wireless spectrum resources are not used to its full potential, and that stands as a barrier before the new techniques applications. So as a new paradigm, CR has been vastly used to enhance the wireless spectrum utilization effectively [1]. SUs have the ability in perceiving and detecting the changes of the dynamic environment for the wireless spectrums, and make the passive spectrum available to the cognitive users. Then SUs can worthily fill the passive spectrum left by the Primary Users (PUs).

In CR networks, PUs and SUs coexist, CR networks provide the capability for the SUs for sharing the wireless channel with the PUs under the interference constraint. There are three modes for CR transmissions: interweaved, overlay and underlay [2]. In the interweaved mode, the SU transmitter implement spectrum sensing to find spectrum holes before it can access the spectrum. The other two modes permit simultaneous SU and PU transmissions. The difference is that the overlay mode presumes perfect PU knowledge at the SU transmitter, whereas the underlay mode presumes a loosely coupled relationship or no cooperation at all between PUs and SUs [2].

As an ideal alternative technology for realizing CR systems, orthogonal frequency division multiplexing (OFDM), due to its adaptability and flexibility with its underlying spectrum shaping capabilities, can improve

*For correspondence significantly the execution of CR systems [3],[4]. IPC is always imposed on each subchannel to guarantee the SUs not causing hurtful interference to the PUs in underlay mode of OFDM-based CR network. So the research of capacity maximization under power control has received lots of interest. The authors [5] proposed a power allocation strategy to optimally maximize the rate of SUs under both the IPC and the rate loss constraint. Authors in [6] maximized the ergodic throughput of a secondary user under the rate loss constraint for primary users by investigating both power allocation and sensing time schemes. Authors [7] considered power allocation in OFDM based underlay CR networks under a given limited transmit power at the SU's transmitter, such that the CR network throughput can be maximized, and the tolerable interference to the PU can be kept within an objective outage probability level. In [8], the authors investigated a power allocation algorithm on the subchannel assignment for downlink OFDM-based cognitive network under both the maximum transmit power constraint and the interference power outage constraint to maximize the utilization of the spectrum and provide proportional fairness to the SUs. In [9], they proposed a new centralized power allocation technique considering departure and arrival of primary users that maximizes total capacity for the cognitive user while regarding an average interference constraint. Authors [10] have proposed suboptimal schemes for subcarrier allocation with associated power loading technique for the OFDM- CR systems. In [11], a power allocation grouping scheme for orthogonal 
frequency division multiplexing (OFDM)-based Cognitive Radio systems is proposed that can improve capacity while the interference power for primary user receivers stays at constant level. Authors in [12], proposed a joint subcarrier and power allocation algorithm for multiuser orthogonal frequency division multiplexing (MU-OFDM) cognitive radio (CR) systems, where the peak power constraint is used to protect the primary user using a proposed lowcomplexity method to update the water level on the water filling algorithm.

The contributions of this paper are as follows. We consider a spectrum underlay CR network where an (MU-OFDM)based CR system coexists with an OFDMA-based primary system. Instead of using the conventional resource allocation method based on IPC and rate loss constraint to protect PU, here we propose a new resource allocation scheme which consider the above constraints but enhances both the fairness and throughput performance of the CR network. The proposed subcarrier allocation algorithm is based on allocating the subcarrier to the best CR user that has the minimum interference channel gain with a proposed fairness constraint, and hence can transmit with a highest power and achieve the utmost throughput while providing improved fairness performance. In addition, we adopt a new power allocation algorithm that will aid the proposed subcarrier allocation algorithm in enhancing the throughput performance.

The remainder of this paper is organized as follows. Section 2 describes the system model and section 3 introduces the proposed resource allocation scheme. Simulation results are proved in section 4 and the conclusions are provided in section 5 .

\section{System model}

\subsection{System architecture and assumptions}

We consider the problem of the resource allocation on downlink of an MU-OFDM secondary system in which the secondary base station is communicating with MSUs using $N$ sub-channel belonging to an OFDMA primary system with $K$ primary users as long as no harmful interference occurs to these PUs. This kind of spectrum sharing is known as underlay sharing. Also, assume the background noise is additive white Gaussian noise (AWGN), and the noise power of each subcarrier is denoted by $N_{0}$.

Let $g_{i}$ be the channel power gain between the primary transmitter (PU-Tx) and receiver (PU-Rx) at subcarrier $i$ (see figure 1), $U_{i}$ be the transmit power of PU allocated to subcarrier $i$, then the transmission rate of $P U_{k}$ (the throughput of $P U_{k}$ ) in the absence of $\mathrm{SU}$ is given by:

$$
R_{k}^{p}=\frac{1}{N} \sum_{i \in L_{k}} \log _{2}\left(1+\frac{g_{i} U_{i}}{N_{0}}\right), \quad \forall k \in\{1,2, \ldots, K\} .
$$

Where $L_{k}$ is the subcarriers set allocated to $P U_{k}$.

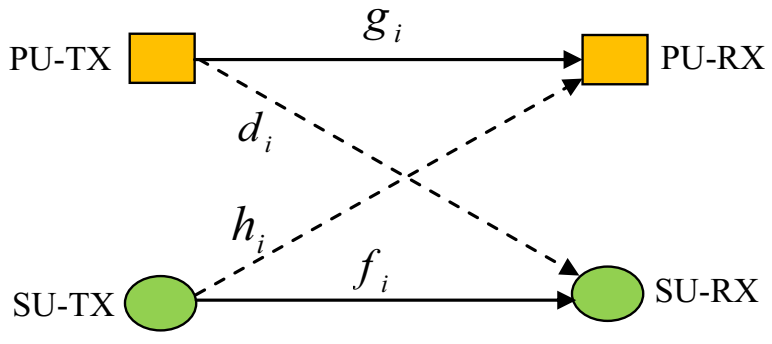

Figure 1. Channel model at subcarrier $i, i \in\{1, \ldots N\}$.

Let $P_{i}$ be the transmit power of SU allocated to subcarrier $i$, and $h_{i}$ be the channel power gain between the secondary transmitter (SU-Tx) and PU's receiver, due to SU's transmission, for any $k \in\{1,2, \ldots, K\}$, then the average rate of $P U_{k}$ (the throughput of $P U_{k}$ ) in the presence of SU is given by:

$$
R_{k}^{s}=\frac{1}{N} \sum_{i \in L_{k}} \log _{2}\left(1+\frac{g_{i} U_{i}}{N_{0}+h_{i} P_{i}}\right) .
$$

Let $f_{i}$ be the channel power gain of the channel between SU's transmitter and receiver at subcarrier $i$, and let $d_{i}$ be the channel power gain of the channel between PU's transmitter and SU's receiver at subcarrier $i$. It is assumed that PU's transmitted signals are Gaussian distributed. Then, the introduced interference to SU by the PU can take the AWGN model with power $J_{i}$, where $J_{i}=d_{i} U_{i}$. The achievable rate of $\mathrm{SU}$ (the throughput of $\mathrm{SU}$ ) is then given by:

$$
r_{s}=\frac{1}{N} \sum_{i=1}^{N} \log _{2}\left(1+\frac{f_{i} P_{i}}{J_{i}+N_{0}}\right) .
$$

The last equation shows the relationship between the number of bits loaded on the subcarrier and the transmit power for a given SU when perfect CSI is available at the transmitter.

\subsection{Interference power and rate loss constraints} [5]

Let $\Gamma_{k}$ be the maximum total interference power that $P U_{k}$ can endure. Then, we can write the constraint of the per user based interference power as

$$
\sum_{i \in L_{k}} h_{i} P_{i} \leq \Gamma_{k}, \quad \forall k \in\{1,2, \ldots, K\} .
$$

Let $\Delta R_{k}$ be the maximum rate loss that PU can endure, then only by satisfying the following constraint, SU's transmission is allowed.

$$
R_{k}^{p}-R_{k}^{s} \leq \Delta R_{k}, \quad \forall k \in\{1,2, \ldots, K\} .
$$

The previous constraints are alluded as the PUs' rate loss constraints. If we define

$$
R_{k} \triangleq R_{k}^{p}-\Delta R_{k}, \quad \forall k \in\{1,2, \ldots, K\} .
$$


then we can rewrite the rate loss constraints as

$$
R_{k}^{s} \geq R_{k}, \quad \forall k \in\{1,2, \ldots, K\}
$$

\section{The proposed resource allocation scheme}

The proposed resource allocation scheme is implemented by two phases. In the first phase, the proposed subcarrier allocation algorithm allocates the subcarrier to the best $\mathrm{m}$ CR user that has the minimum interference channel gain $h_{i}$, with a proposed fairness constraint to achieve a maximum throughput under IPC over that subcarrier.

The steps of the proposed subcarrier allocation algorithm are as follows:

- Interference channel gains information for all $\mathrm{N}$ subcarriers are centrally collected from all CR users.

- Find the minimum interference channel gain detected for each subcarrier.

- Arrange all the subcarriers ascendingly according to the interference channel gain defined on the previous step.

- Set a fairness constraint threshold $\eta \cdot N_{\text {avg }}$ which define the maximum number of subcarriers that can be allocated to any $\mathrm{CR}$ user during the subcarrier allocation process to enhance the fairness performance.

Where:

$$
N_{\text {avg }}=N / M
$$

and $\eta$ is a fairness controlled parameter which is equal to 1 $(\eta=1)$ for a fair subcarrier allocation and greater than 1 $(\eta>1)$ for a partial fair subcarrier allocation.

The relationship between the rate loss constraint and the per subcarrier based IPC [5] is satisfied when

$$
\tilde{\Gamma}_{k}=N_{0}\left(2^{K \Delta R_{k} /\left|L_{k}\right|}-1\right)
$$

where $\tilde{\Gamma}_{k}$ is the maximum interference power that each subcarrier of $P U_{k}$ can endure and $\Delta R_{k}$ is the maximum rate loss that can be endured by the PU where the relationship between the PU throughput and its SNR is shown in equations (1) and (2).

- The CR system selects the best CR user that has the minimum interference channel gain $h_{i}$ for each subcarrier $i, i \in\{1, \ldots, N\}$ with the fairness constraint threshold $\eta \cdot N_{\text {avg }}$ defined previously. From the IPC condition shown next in equation (10), we define the transmitted power $P_{i}$ that every SU can send over each subcarrier $i$

$$
h_{i} P_{i} \leq \Gamma_{k}, \quad \forall i \in L_{k}
$$

Choosing a minimum interference channel gain lead to maximize the value of transmitted power $P_{i}$ and then enhance the throughput of the SU according to equation (3).

All the previous steps are summarized in figure 2.

In the second phase, a new power allocation algorithm is proposed to efficiently manage and distribute the power budget of each SU over its allocated subcarriers.

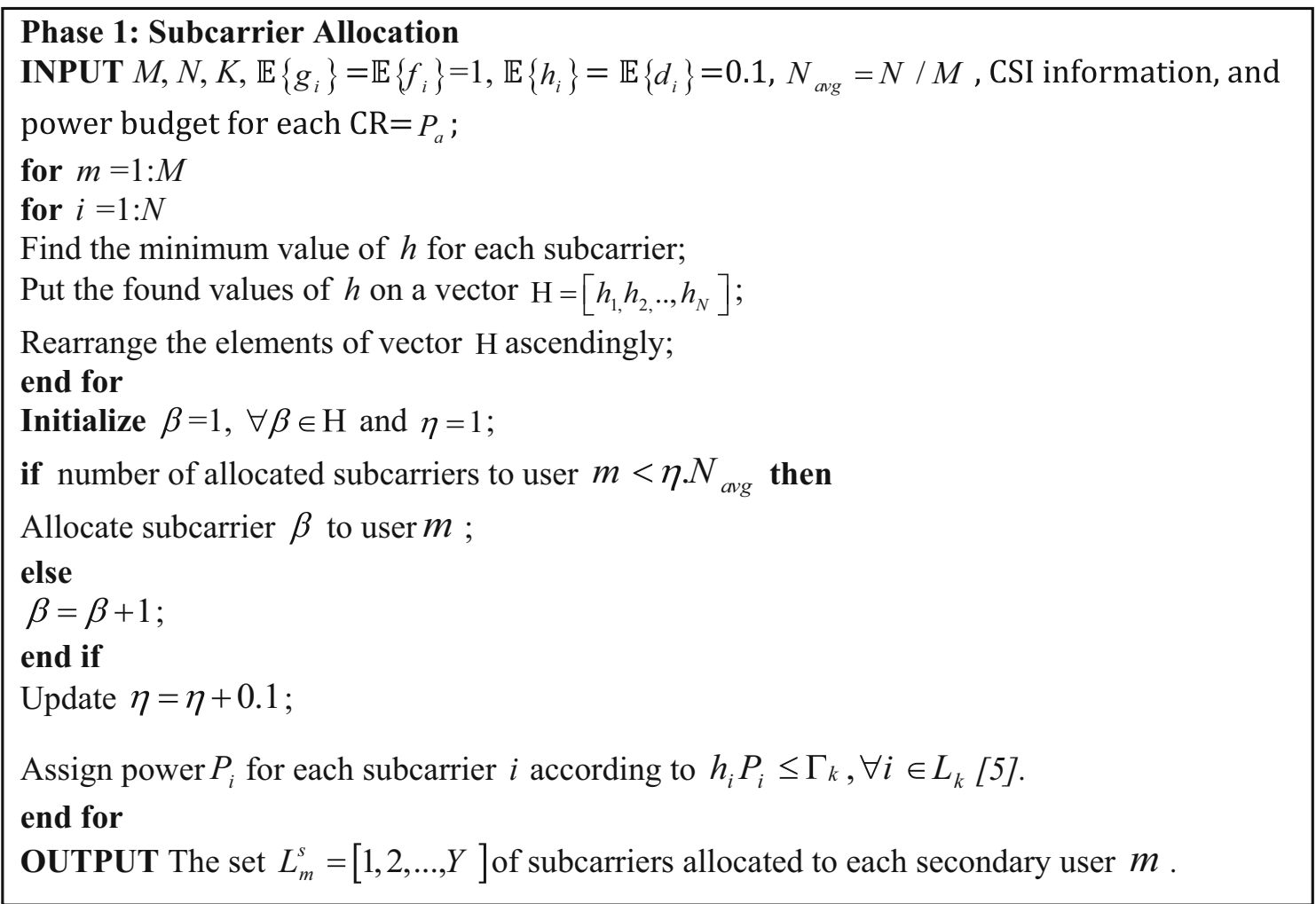

Figure 2. The proposed subcarrier allocation algorithm. 


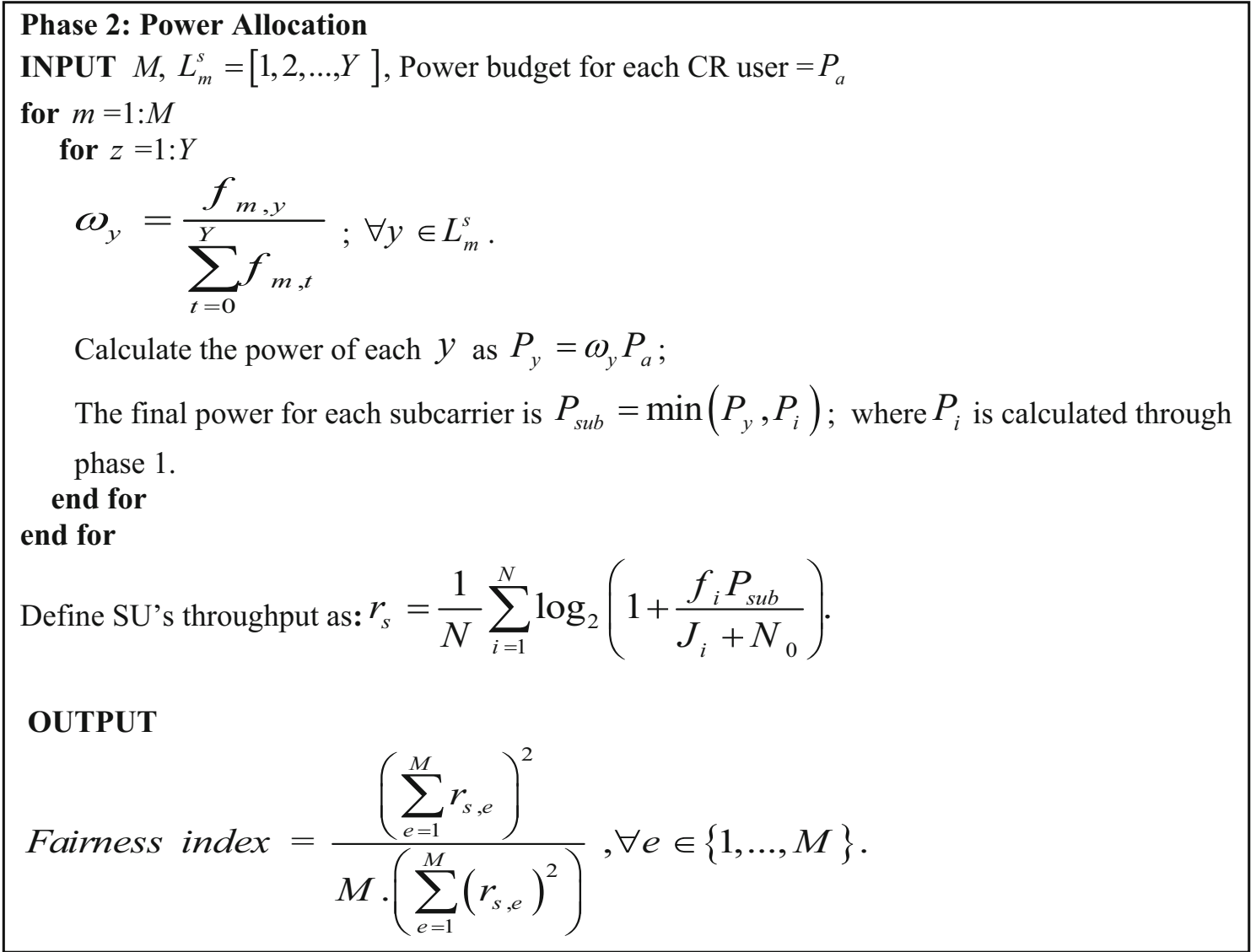

Figure 3. The proposed power allocation algorithm.

We suppose that each SU has been allocated to a set $L_{m}^{s}$ of subcarriers. Assume the maximum number of subcarriers inside this set is denoted by $\mathrm{Y}$.

- The amount of power that will be allocated to each subcarrier on the set $L_{m}^{s}$ depends on the direct channel gain for the $\mathrm{SU}$ over this subcarrier to enhance the total CR network throughput.

- Let the power budget for every $S U_{m}$ is $P_{a}$, then the allocated power per subcarrier y will be $P_{y}=\omega_{y} P_{a}$

where

$$
\omega_{y}=\frac{f_{m, y}}{\sum_{t=0}^{Y} f_{m, t}} ; \quad \forall y \in L_{m}^{s}
$$

Where $L_{m}^{s}$ is the subcarriers set allocated to each SU.

Thus, the weight $\omega_{y}$ can be defined as the ratio of SU's direct channel gain for the current subcarrier y to the sum of the same SU's direct channel gains for all the set of subcarriers allocated to that $\mathrm{SU}$.

- Finally, with applying the IPC constraint, the allocated subcarrier power will be $P_{s u b}=\min \left(P_{y}, P_{i}\right)$ for each subcarrier and the equation of the throughput achieved by the $\mathrm{SU}$ in equation (3) can be rewritten as

$$
r_{s}=\frac{1}{N} \sum_{i=1}^{N} \log _{2}\left(1+\frac{f_{i} P_{\text {sub }}}{J_{i}+N_{0}}\right) .
$$

The fairness index that measure the fairness of the throughput distribution among the secondary users can be calculated as

$$
\text { Fairness index }=\frac{\left(\sum_{e=1}^{M} r_{s, e}\right)^{2}}{M \cdot\left(\sum_{e=1}^{M}\left(r_{s, e}\right)^{2}\right)}, \quad \forall e \in\{1, \ldots, M\} .
$$

Where $r_{s}$ is the SU throughput.

All the previous steps are summarized in figure 3 .

\section{Simulation results}

\subsection{Performance of the proposed scheme under perfect channel state information}

In this section, several examples are displayed to verify the effectiveness of the proposed resource allocation strategy. In these simulation examples, we assume that all the 
included channels (i.e., the secondary link, the primary link, and the interference links) are Rayleigh distributed. So, the channel power gains of these channels are exponentially distributed. MATLAB ${ }^{\circledR}$ is used to develop the simulation program for the proposed scheme, and all the CR network parameters and equations are considered on the developed MATLAB code to assess the performance of the proposed scheme compared to the conventional IPC based scheme. Where the conventional IPC based scheme proposed in [5], illustrates a power allocation algorithm with random subcarrier allocation under a new primary transmission protection criteria. The simulation results are displayed in this part are taken out by averaging over $10^{6}$ independent simulation runs. The average channel power gains for the secondary link $f_{i}$ and the primary link $g_{i}$ are presumed to be 1, i.e., $\mathbb{E}\left\{g_{i}\right\}=\mathbb{E}\left\{f_{i}\right\}=1, \forall_{i}$. The average channel power gain for the interference links is presumed to be 0.1, i.e.,

Table 1. The description of the CR network parameters.

\begin{tabular}{lc}
\hline Parameter & Value or description \\
\hline Channel model & Rayleigh distributed \\
Mean of $g_{i}$ & 1 \\
Mean of $f_{i}$ & 1 \\
Mean of $d_{i} \& h_{i}$ & 0.1 \\
No. of primary users K & 1 \\
No. of secondary users M & $4,8,16$ \\
No. of subcarriers N & 128 \\
Primary user power budget & $1-30 \mathrm{~dB}$ \\
Secondary user power budget & $20 \mathrm{~dB}$ \\
Maximum rate loss of primary user & $10 \%$ \\
\hline
\end{tabular}

$\mathbb{E}\left\{h_{i}\right\}=\left\{d_{i}\right\}=0.1, \forall_{i}$. Moreover, we presume that the system contains a single primary user and multi-secondary users. The PU shares 128 subcarriers with SNR changes from 1 to $30 \mathrm{~dB}$. It is also presumed that the primary system adopts equal power allocation over its subcarriers. The noise power on each subcarrier is presumed to be identical, and equal to 1 , i.e., $N_{o}=1$. The cognitive radio network simulation parameters are summarized in table 1 .

Figure 4 shows the achieved throughput by the secondary network as a function of the SNR received from the primary user at secondary power budget $P_{a}=20 \mathrm{~dB}, 10 \%$ PU rate loss, $\eta=1.2$, and various number of SUs. As expected, the throughput of the secondary network significantly decreases as the PU's SNR becomes higher. The reason behind is that the significant increase of the interference produced by the high PU's power on the SU as in equation (3). The figure also reveals that the secondary network throughput achieved by the proposed scheme is significantly higher than that of the conventional scheme and it significantly increases as the number of SUs increases. This is because, as the number of SUs increases, the opportunity that the selected SU has a low interference channel gain is improved which improves the efficiency of the proposed algorithm on allocating the subcarrier to the best SU.

The throughput of the primary network as a function of PU's SNR is presented in figure 5. It is observed that the proposed scheme maintains the PU's QoS as the conventional scheme by allowing the primary network to achieve the same throughput. This means that the proposed scheme does not produce a harmless interference on the PU since it allocates the subcarriers to the SU that has the

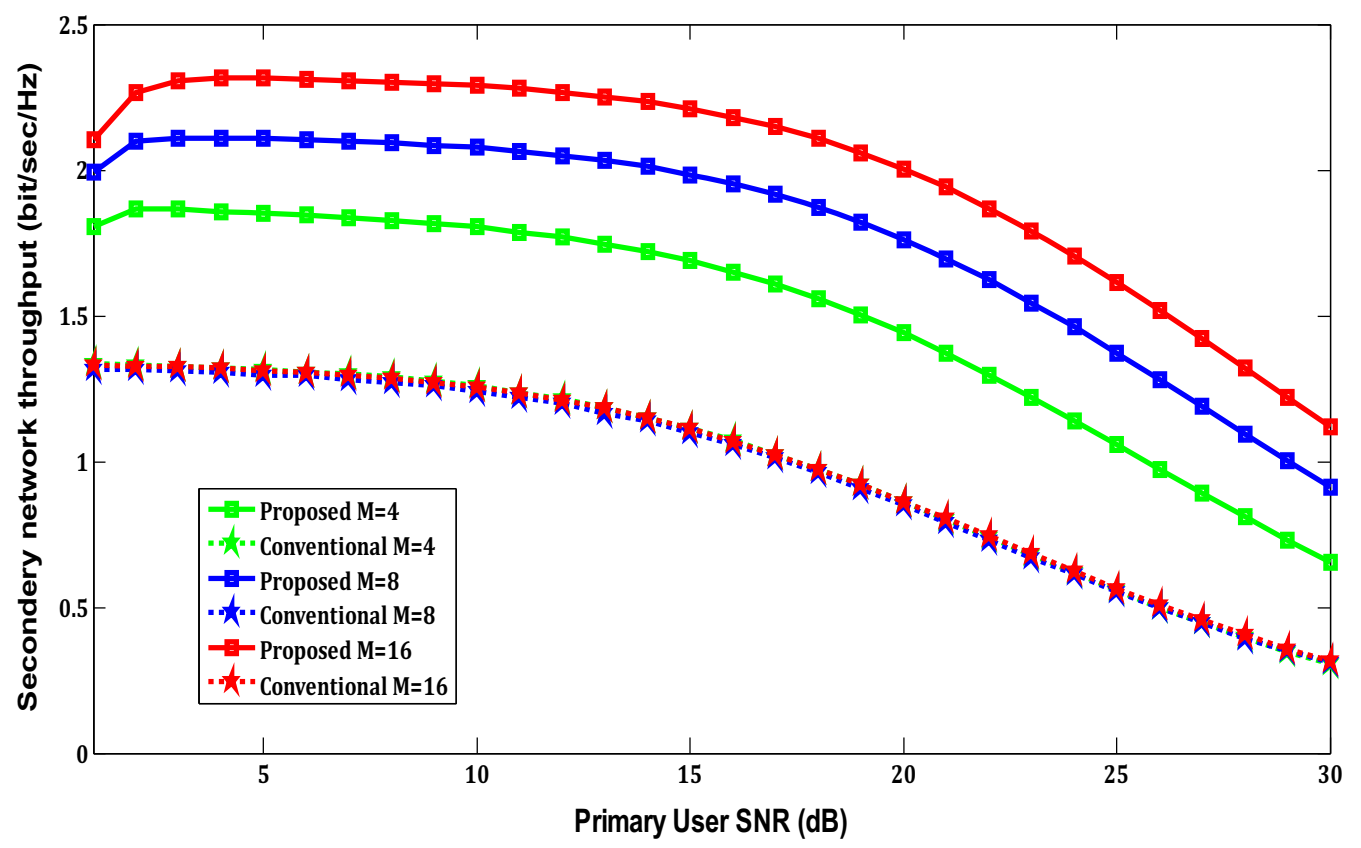

Figure 4. The secondary network throughput (bit/sec/Hz) versus the PU SNR (dB) at $\eta=1.2$ with different numbers of SUs. 


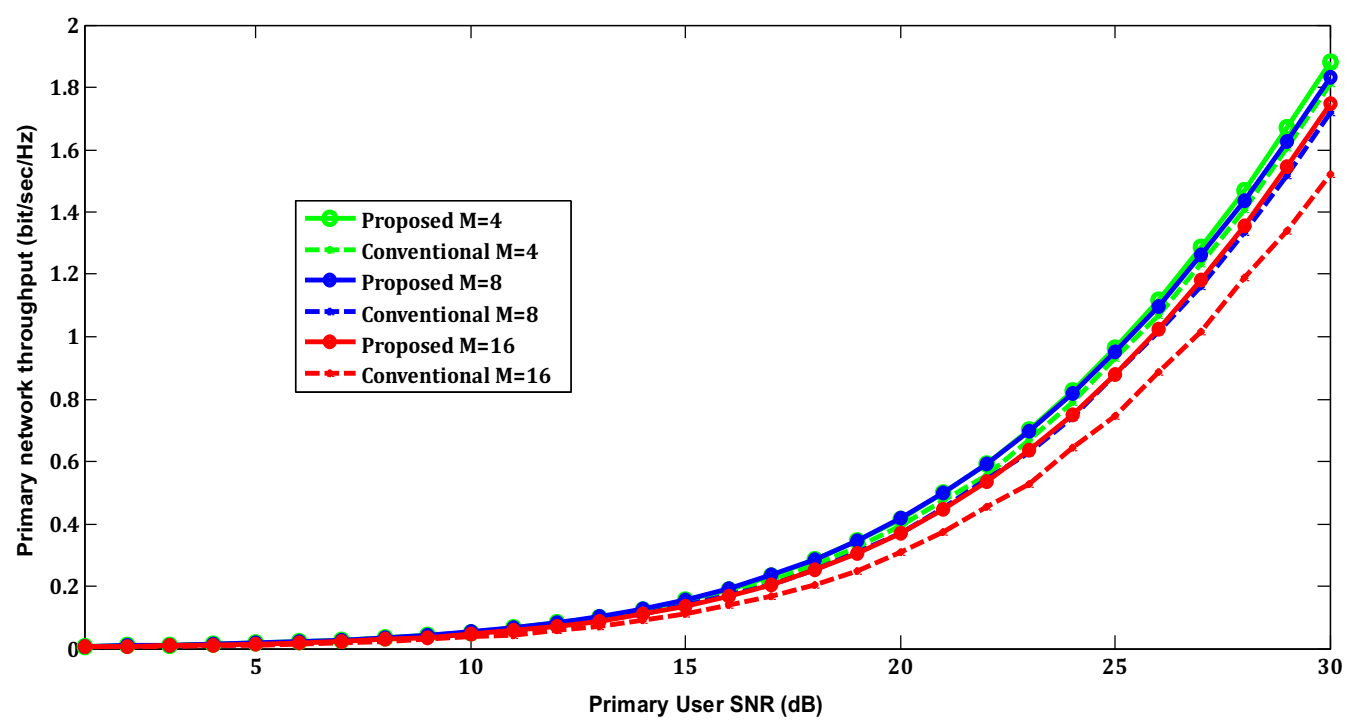

Figure 5. The primary network throughput (bit/sec/Hz) versus the PU SNR (dB) at $\eta=1.2$ and different numbers of SUs.

minimum interference channel gain (i.e., the lowest interference effect on PU).

Figure 6 presents the fairness performance of the proposed resource allocation scheme as a function of PU's SNR at a $\eta=1.2$. As observed from figure, the fairness index slightly decreases as the PU's SNR increases as seen in equations (12), (13). Also, the fairness index increases with decreasing the number of SUs, because the average number of subcarriers allocated to each SU, $N_{\text {avg }}$ under the same total subcarriers $N$ will be increased. This will give the opportunity to each individual SU to experience different subcarrier interference channel gains and hence, improving the fairness performance. In addition, the proposed scheme achieves a significantly high fairness index which is slightly lower than that of the conventional scheme. In the conventional scheme, the number of

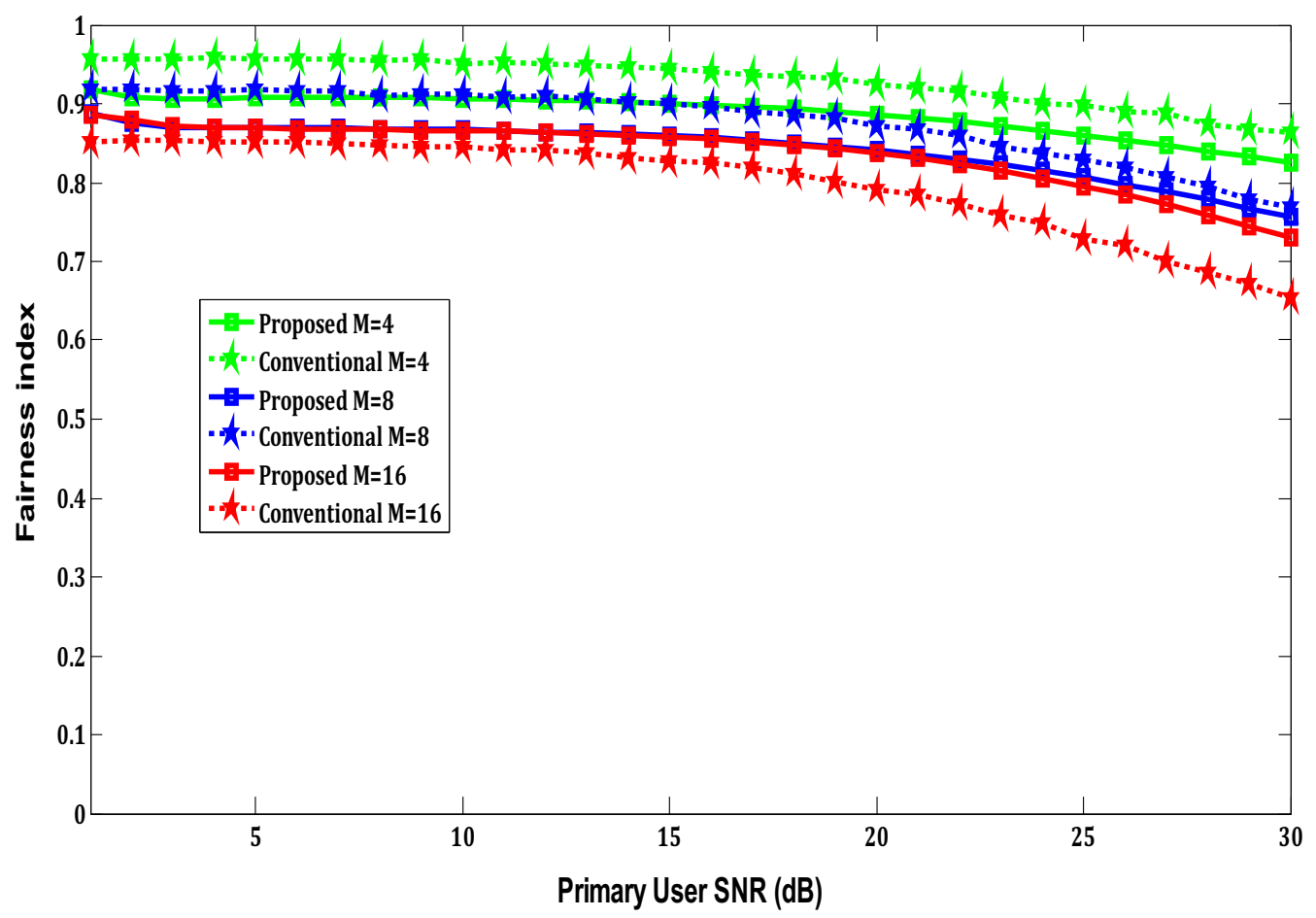

Figure 6. Fairness index for CRs with the primary user SNR (dB) at SU power budget $P_{a}=20 \mathrm{~dB}, \eta=1.2$ and different number of SUs. 


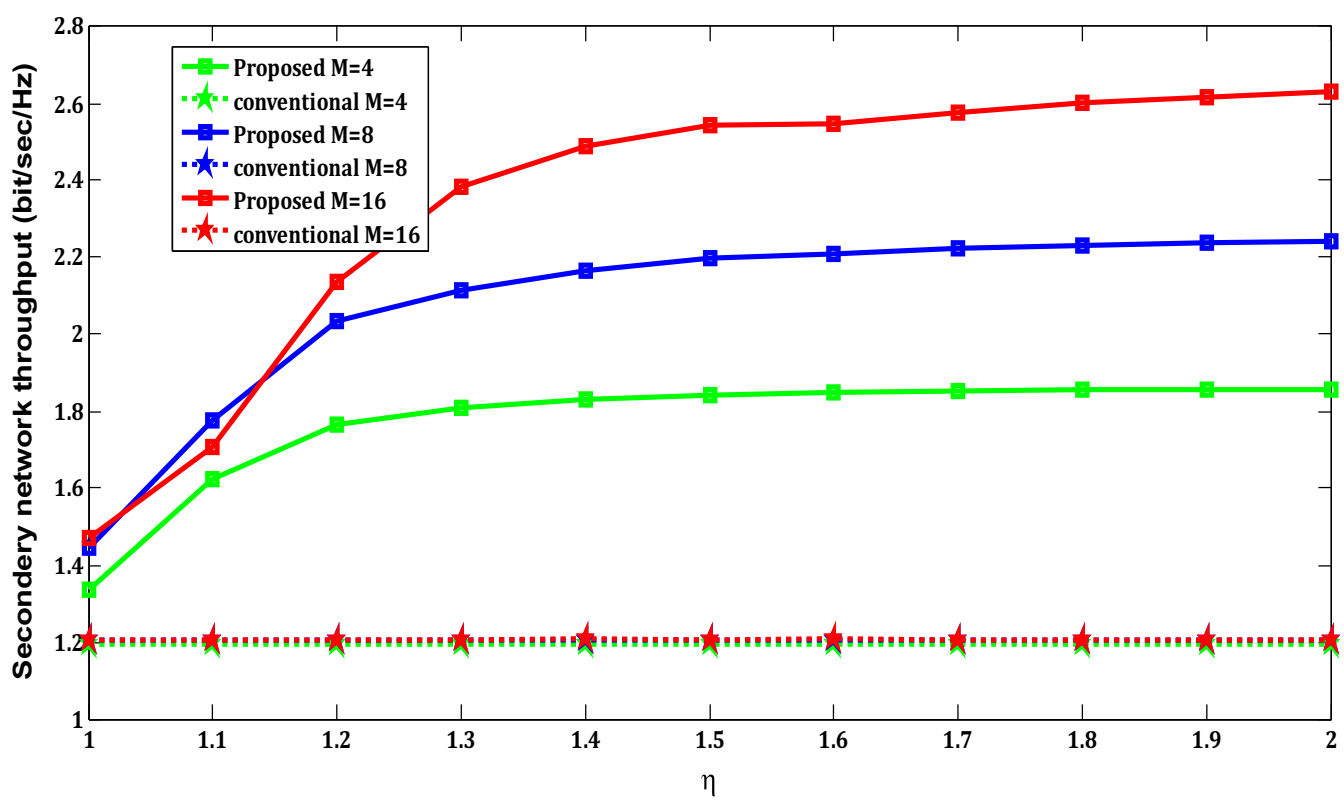

Figure 7. The secondary network throughput (bit/sec/Hz) versus $\eta$ at SU power budget $P_{a}=20 \mathrm{~dB}$ and different numbers of SUs.

allocated subcarriers per SU is approximately equal to $N_{a v g}$, which leads to its good fairness performance, and this case is equivalent to $\eta=1$ for the proposed scheme.

Figure 7 presents the relation between $\eta$ and the $\mathrm{CR}$ network throughput at PU's $\mathrm{SNR}=2 \mathrm{~dB}$. The figure shows that the $\mathrm{CR}$ throughput of the proposed scheme significantly increases with the increase of $\eta$ and when $\eta$ becomes greater than 1.4, the CR throughput is approximately saturated to its maximum values with no significant increase. This is because as $\eta$ increases, the number of good subcarriers that can be allocated to a specific SUs will be larger than $N_{\text {avg }}$ which enable the proposed scheme to efficiently allocate the subcarriers to the best SUs which can have the minimum interference channel gain under a more relaxed fairness constraints.

Figure 8 shows the effect of changing $\eta$ on the fairness index of the CR network at PU's $\mathrm{SNR}=2 \mathrm{~dB}$. It is observed that at $\eta=1$, the fairness index of the proposed scheme is approximately the same as that of the conventional scheme. In addition, the fairness index of the proposed scheme slightly decreases as $\eta$ increases $(\eta>1)$ because the number of allocated subcarriers to each $\mathrm{CR}$ user will not be the same. So, some SUs have more allocated subcarriers than the others which slightly reduces the fairness index.

Table 2 gives a comparison between the proposed scheme with, without applying fairness constraint and the conventional scheme. The results reflect that the throughput of the secondary network achieved by the proposed scheme with applying fairness constraint scheme at $\eta=1.3$ approximately converges to the maximum values achieved without applying fairness constraint and significantly higher than the conventional one. On the other hand, the proposed scheme with applying fairness constraint scheme at $\eta=1.3$ provides a significantly enhanced fairness performance which approximately converges with that of the conventional scheme and significantly higher than that of the proposed scheme without applying fairness constraint.

\subsection{Performance of the proposed scheme under imperfect channel state information}

Most existing works assume that $h_{i}$ is perfectly known. However, $h_{i}$ is imperfect, which is an important scenario for CR systems. A channel estimation is done by (SU-Rx) to estimate the SU-PU interference channel gain, denoted by $h_{i}$. Existence of channel estimation errors is assumed, i.e.,

$$
h_{i}=\hat{h}_{i}+e_{E s t}, \quad \forall i \in\{1,2, \ldots N\}
$$

where $\hat{h}_{i}$ is the estimated channel gain and $e_{E S T}$ is the estimation error. Furthermore, we assume that estimation error $e_{E S T}$ is a uniform random distribution vector with zero-mean and variance $\sigma_{e}^{2}$ which is assumed here to be 0.5 .

Figure 9 shows the achieved throughput by the secondary network as a function of the received SNR from the primary user at secondary power budget $P_{a}=20 \mathrm{~dB}, 10 \%$ primary rate loss, $\eta=1.2$, and $\mathrm{M}=8$ SUs. As expected, CR's throughput significantly decreases as PU's SNR 


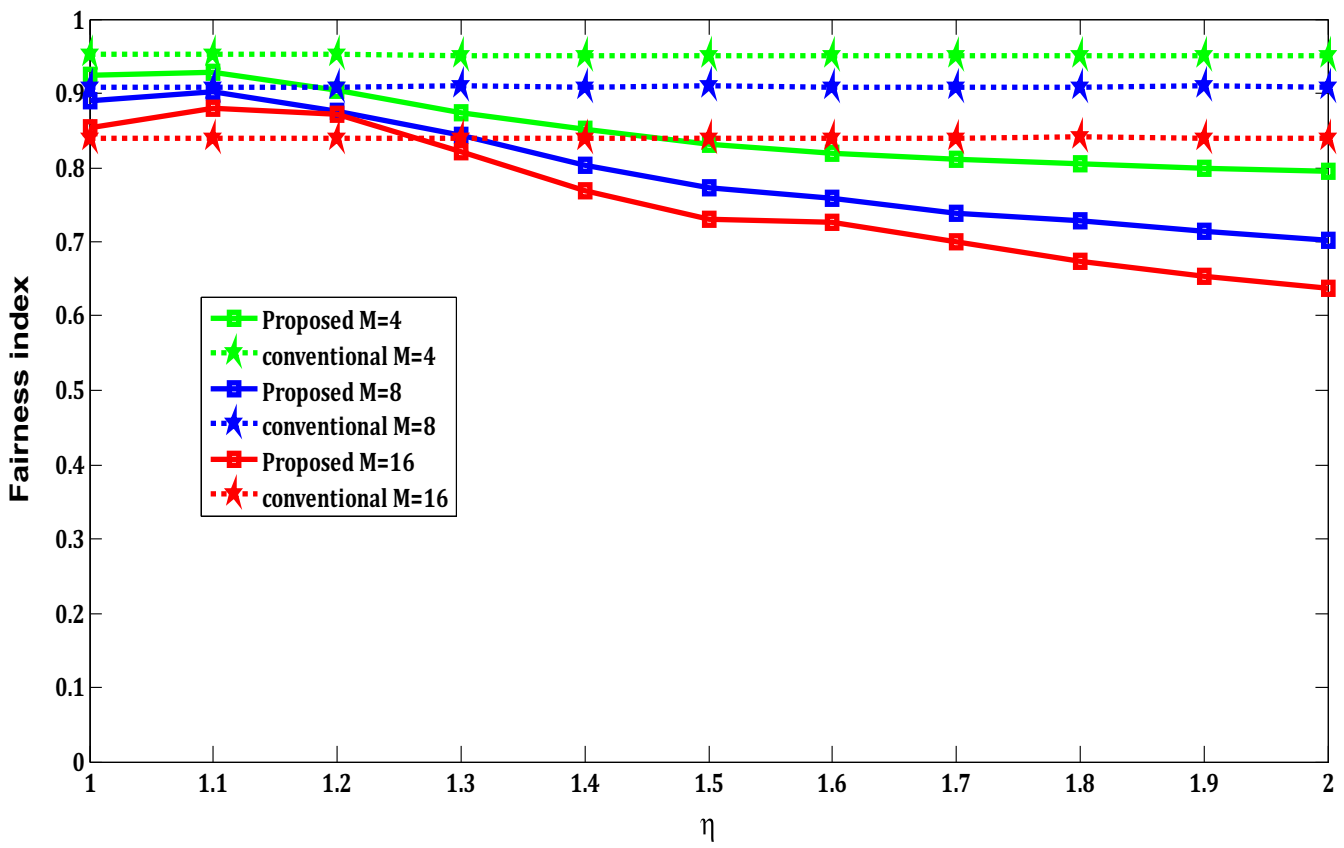

Figure 8. The fairness index in CR network versus $\eta$ at SU power budget $P_{a}=20 \mathrm{~dB}$, and different numbers of SUs.

Table 2. The throughput of the secondary network by the proposed scheme with applying fairness constraint scheme at $\eta=1.3$, without applying fairness constraint and finally for the conventional scheme at $\mathrm{M}=16 \mathrm{CR}$.

\begin{tabular}{rcc}
\hline Different schemes & Throughput (bit/sec/Hz) & Fairness index \\
\hline Conventional scheme & 1.3104 & 0.8411 \\
Proposed scheme without fairness constraint & 2.6692 & 0.6353 \\
Proposed scheme with fairness constraint at $\eta=1.3$ & 2.4403 & 0.8283
\end{tabular}

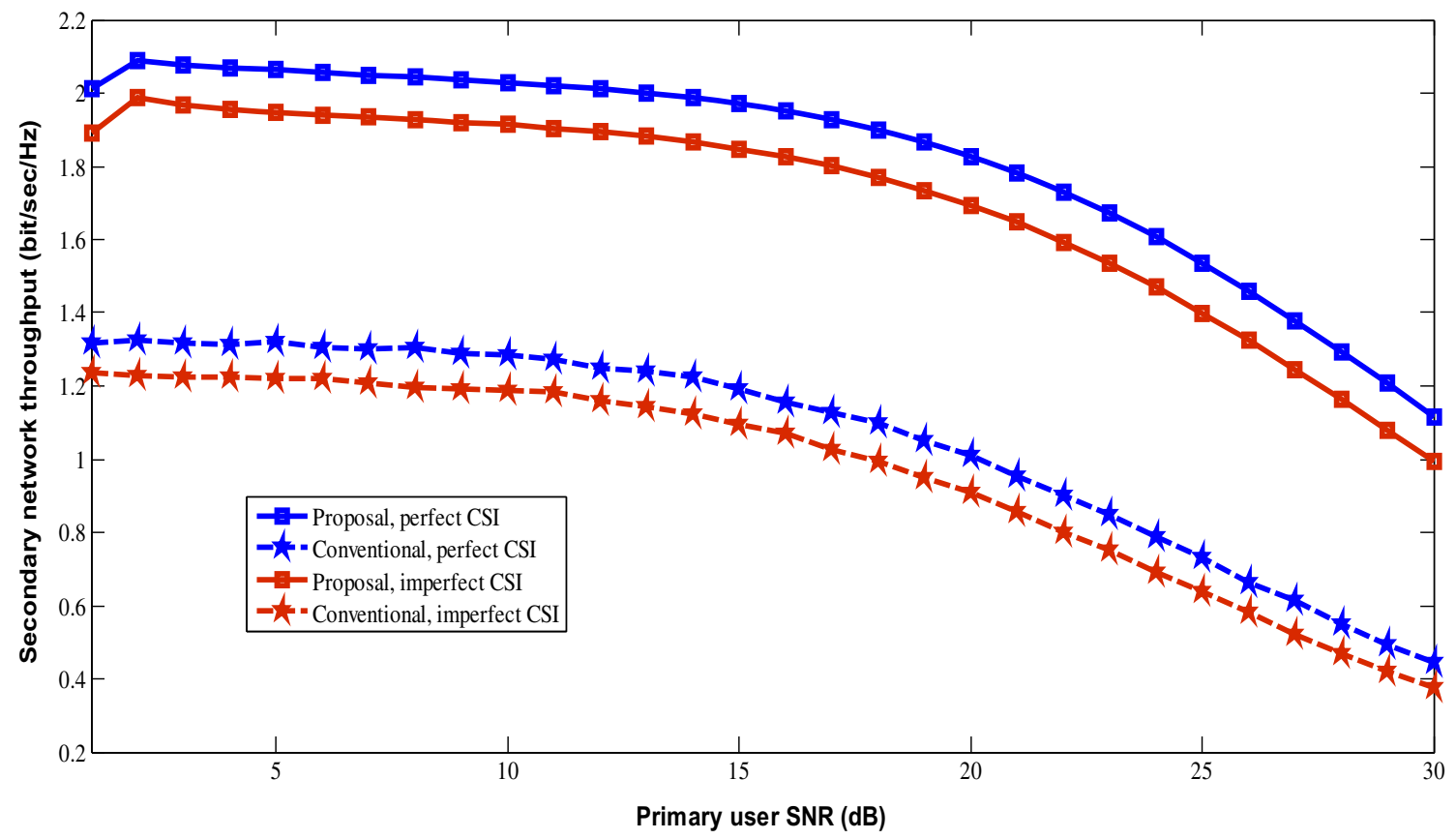

Figure 9. The secondary network throughput (bit/sec/Hz) versus the PU SNR $(\mathrm{dB})$ at $\eta=1.2$ with $\mathrm{M}=8 \mathrm{SUs}$ under both perfect and imperfect CSI. 
increases due to the significant increase of the interference produced by the high PU's power on the SU as in equation (3). The figure also demonstrates that the degradation occurred in the throughput due to the imperfect CSI is small. This reflects the robustness of the proposed scheme in the real environment.

Figure 10 draws the relation between $\eta$ and the $\mathrm{CR}$ network throughput at PU's $\mathrm{SNR}=12 \mathrm{~dB}$. The

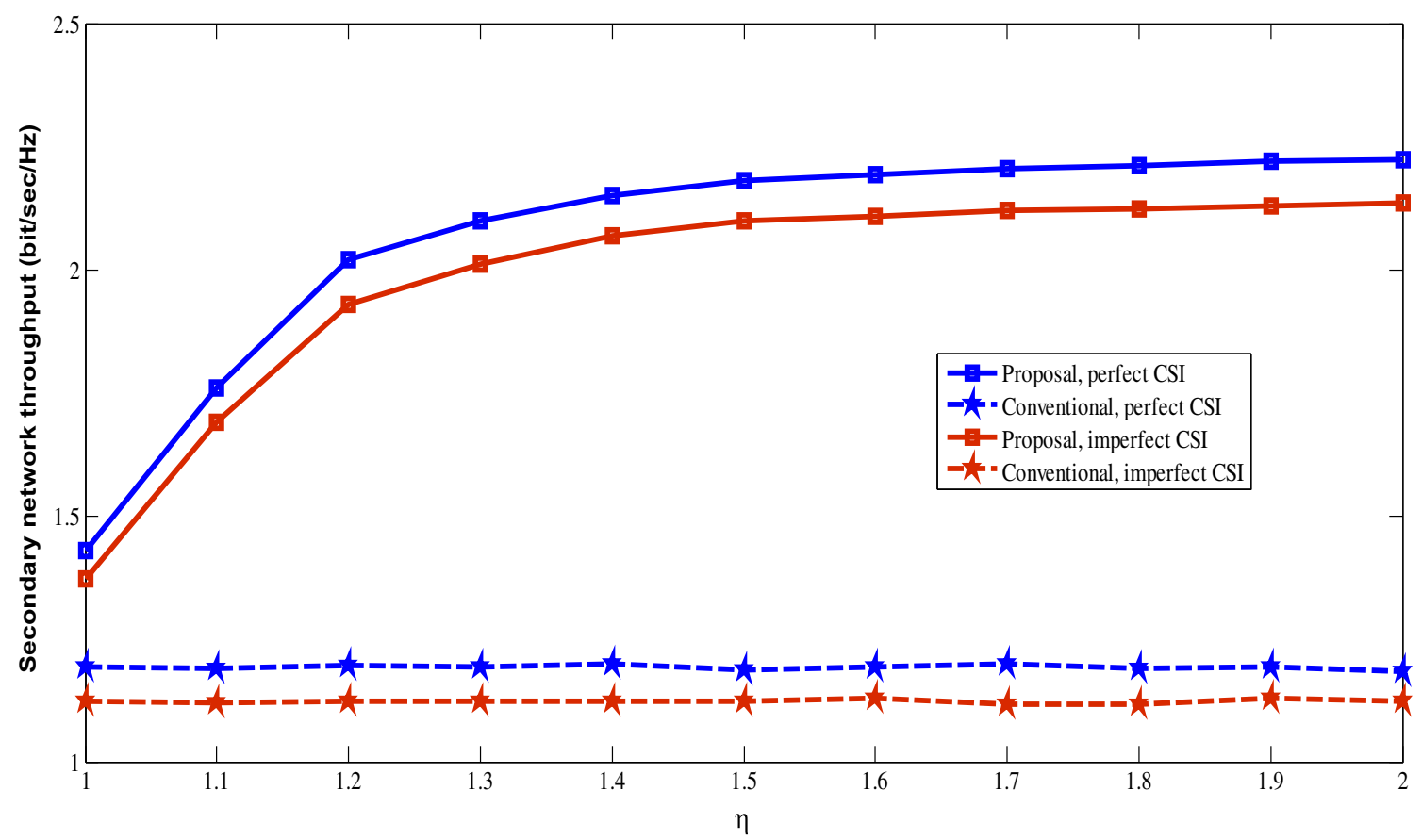

Figure 10. The secondary network throughput (bit/sec/Hz) versus $\eta$ at SU power budget $P_{a}=20 \mathrm{~dB}$ and $M=8$ under both perfect and imperfect CSI.

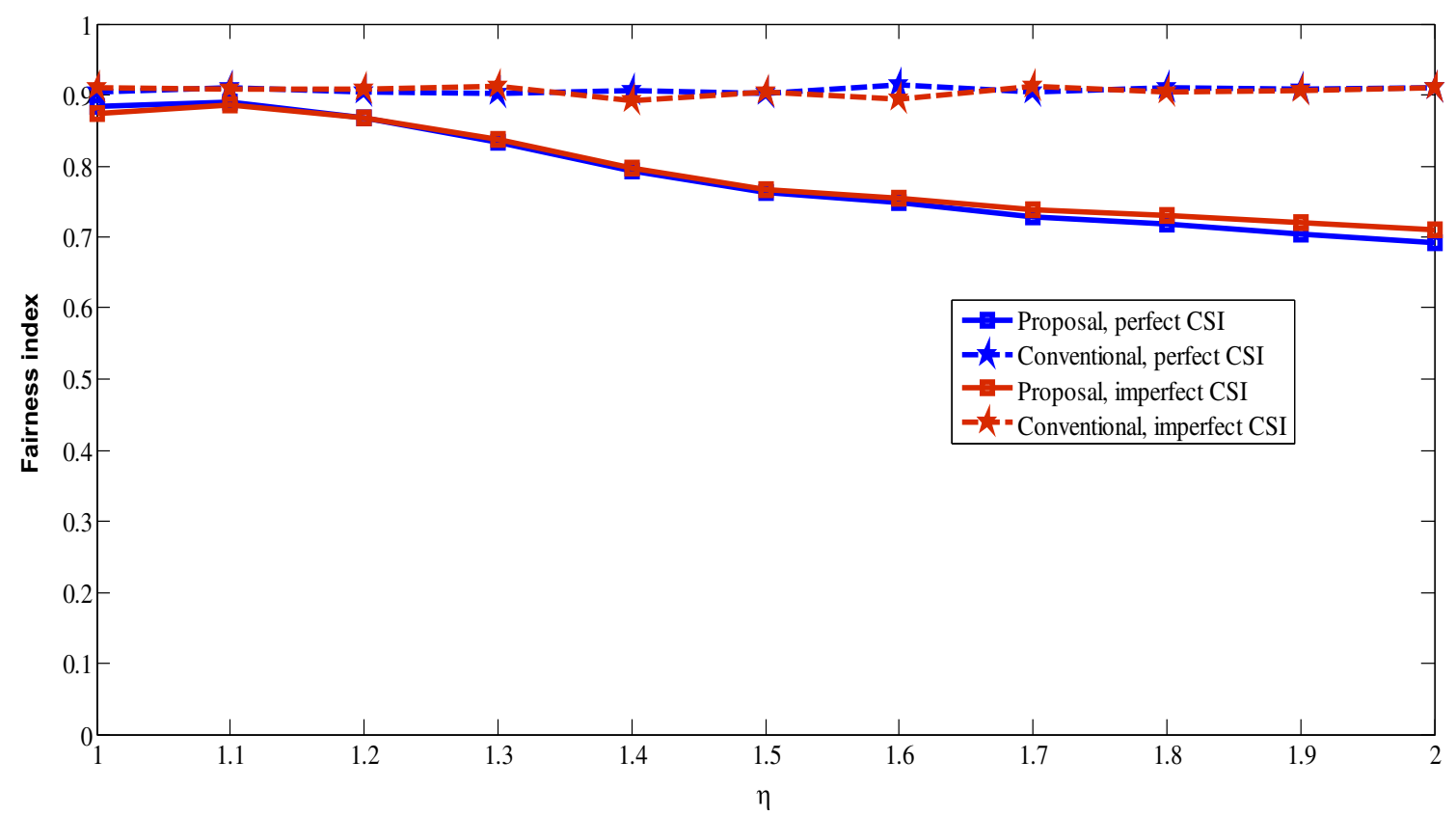

Figure 11. The fairness index in CR network versus $\eta$ under perfect and imperfect CSI. 
figure shows that the CR throughput by the proposed resource allocation scheme significantly increases with increasing $\eta$. When $\eta$ becomes $\geq 1.4$, the CR throughput is approximately saturated to its maximum values. The figure also demonstrates that the proposed scheme significantly outperforms the conventional scheme especially for $\eta \geq 1$.4. The degradation on the proposed scheme due to the imperfect channel statement is small especially for the usable range of the fairness controlled parameter $(\eta<1.4)$.

Figure 11 shows the effect of changing $\eta$ on the fairness index of the CR network at PU's SNR $=12 \mathrm{~dB}$. It is observed that the fairness index of the proposed scheme is slightly lower than that of the conventional scheme and slightly decreases as $\eta$ increases. It is worth to mention that the proposed scheme achieves its maximum throughput at $\eta=1.4$ which means that a slight degradation in the fairness index is expected. In addition, it is clear that the imperfect CSI has no effect on the fairness performance of both schemes.

\section{Conclusion}

The proposed centralized subcarrier and power allocation scheme for underlay MU-OFDM in this paper efficiently distributes the available subcarriers among cognitive users and enhances both the fairness and the throughput performance of the cognitive network while maintaining the QoS of primary users. Simulation results reflect that the proposed scheme achieved a significantly higher CR network throughput than that of the conventional IPC based schemes and provides significantly enhanced fairness performance. Through applying the fairness constraint, the proposed algorithm is still robust in enhancing the throughput performance, while achieving a significant high fairness performance. Also, to the contrary of the conventional IPC based schemes, the proposed algorithm is able to significantly increase the achieved throughput as the number of CR users increases. In addition, the proposed scheme proved its robustness through both the perfect and imperfect channel state information.

\section{References}

[1] Simon H S 2005 Brain-empowered wireless communications. IEEE J. Sel. Areas Commun. 23: 201-220

[2] Srinivasa S and Jafar S A 2007 The throughput potential of cognitive radio: a theoretical perspective. IEEE Commun. Mag. 45(5): 73-79

[3] Weiss T and Jondral F K 2004 Spectrum pooling: an innovative strategy for the enhancement of spectrum efficiency. IEEE Communication Mag. 43(3): S8-S14

[4] Mahmoud H A, Yucek T and Arslan H 2009 OFDM for Cognitive Radio: Merits and Challenges. IEEE Wireless Communications. 16(2): $6-15$

[5] Kang X, Garg H K, Liang Y and Zhang R 2010 Optimal power allocation for OFDM-based cognitive radio with new primary transmission protection criteria. IEEE Trans. Wirel. Commun. 9(6): 2066-2075

[6] Ning B, Yang S, Liu L and Lu Y 2014 Resource allocation for OFDM cognitive radio with enhanced primary transmission protection. IEEE Commun. Lett. 18(11): 2027-2030

[7] Zhou X, Wu B, Ho P and Ling X 2011 An Efficient Power Allocation Algorithm for OFDM Based Underlay Cognitive Radio Networks. In: IEEE Communications Society subject matter experts for publication in the IEEE Globecom proceedings.

[8] Li J, He C and Jiang L 2013 An proportional fair resource allocation in OFDM-based cognitive radio networks under imperfect channel-state information. In: IEEE Wireless Communications and Networking Conference (WCNC). 1814-1818

[9] Kulkarni K and Banerjee A 2013 Power allocation for OFDM-based cognitive radio systems under average interference constraint. In: National Conference on Communications (NCC), IEEE. 1-5

[10] Chakraborty S, Dhanuka P, Kumar A and Maity S P 2013 Subcarrier and power allocation schemes for multiuser OFDM-based cognitive radio systems. IEEE. 1-5

[11] Hosseini E and Falahati A 2013 Power allocation grouping scheme considering constraints in two separate stages for OFDM-based cognitive radio system. In: International Conference on Electrical Information and Communication Technology (EICT). 1-6

[12] Chen C and Wang C 2010 Joint subcarrier and power allocation in multiuser OFDM-based cognitive radio systems. In: IEEE Communications Society subject matter experts for publication in the IEEE ICC. 1-5 